study showed a significant decrease in mean arterial BP from baseline; this effect was driven by a decrease in mean systolic BP $(-15 \mathrm{mmHg}$ after 5 weeks of treatment in the first study, $-16 \mathrm{mmHg}$ after 3 weeks of treatment in the $400 \mathrm{mg} /$ day group; $P=0.04$ for both). No significant change in diastolic BP or heart rate was seen in any group. Endothelial function, as measured by flow-mediated vasodilation of the brachial artery, significantly improved in all groups except the $200 \mathrm{mg} /$ day group. The authors note that tetrahydrobiopterin was well-tolerated.

Original article Porkert M et al. (2008) Tetrahydrobiopterin: a novel antihypertensive therapy. J Hum Hypertens 22: 401-407

\section{A single measurement of urinary NGAL can identify acute kidney injury}

Serum creatinine levels rise several days after acute kidney injury but are an insensitive marker of kidney function. Urinary levels of neutrophil gelatinase-associated lipocalin (NGAL) correlate with the severity of ongoing renal tubular damage and represent one possible alternative marker. Studies of NGAL conducted to date in the setting of acute kidney injury have generally involved patients with injury of a specific cause and known timing.

Nickolas and colleagues' prospective study included 635 patients (mean age 60 years) admitted to a hospital emergency room. Final diagnoses included 30 patients with acute kidney injury, 88 with prerenal azotemia, and 106 with nonprogressive chronic kidney disease; the other 411 patients had normal kidney function. NGAL and other biomarkers (creatinine, $a_{1}$-microglobulin, $a_{1}$-acid glycoprotein and $\mathrm{N}$-acetyl- $\beta$-D-glucosaminidase) were assessed in patients' samples of blood and urine obtained at admission.

In this heterogeneous cohort of patients, NGAL was the most useful biomarker. Only patients diagnosed on the basis of clinical criteria as having acute kidney injury had significantly elevated urinary levels of NGAL at admission, regardless of the mechanism of injury. Maximal sensitivity and specificity of NGAL was achieved with a cutoff of $130 \mu \mathrm{g} / \mathrm{g}$ creatinine; levels above this threshold predicted excess morbidity and poor outcomes. NGAL levels were a better predictor of outcome than the other markers of acute kidney injury tested and remained highly diagnostic even when the time of injury was unknown.

Original article Nickolas TL et al. (2008) Sensitivity and specificity of a single emergency department measurement of urinary neutrophil gelatinase-associated lipocalin for diagnosing acute kidney injury. Ann Intern Med 148: 810-819

\section{Should femoral catheterization for acute RRT be avoided to prevent infection?}

Jugular vein catheters are thought to be less prone to infection than femoral vein catheters, but evidence from randomized trials has been lacking. Now, results of the Cathedia study in France suggest that there is no significant difference in the rate of infection between jugular and femoral access in critically ill patients requiring acute renal replacement therapy (RRT).

Parienti et al. conducted a multicenter, prospective, evaluator-blinded trial in which 750 adults were randomly allocated to undergo femoral vein or jugular temporary vein catheterization for short-term vascular access for RRT. The incidence of catheter colonizations per 1,000 catheter days was $40.8(95 \% \mathrm{Cl} 29.3-55.4)$ in the femoral group and 35.7 (95\% Cl 25.0-49.5) in the jugular group $(P=0.54)$. Femoral catheterization was associated with $1.5(95 \% \mathrm{Cl}$ $0.1-6.4)$ bloodstream infections per 1,000 days compared with $2.3(95 \% \mathrm{Cl} 0.3-7.7)$ for jugular catheteriztion $(P=0.42)$. The risk of catheter colonization increased with BMI in the femoral group, but not in the jugular group. Jugular insertion, mainly by use of the landmark technique, had a higher rate of mechanical complications than femoral insertion. The rate of hematoma formation was $3.6 \%$ in the jugular group compared with $1.1 \%$ in the femoral group $(P=0.03)$.

The authors suggest that jugular catheterization should be strongly considered for patients with a high BMI who require acute RRT, but that femoral catheterization by an experienced operator might reduce the likelihood of mechanical complications compared with jugular catheterization in nonobese, bedbound, critically ill patients, without increasing the risk of infection.

Original article Parienti JJ et al. (2008) Femoral vs jugular venous catheterization and risk of nosocomial events in adults requiring acute renal replacement therapy: a randomized controlled trial. JAMA 299: 2413-2422 